\title{
Estimación de la ganancia genética esperada de Pinus tecunumanii en plantaciones forestales en Oxapampa, Perú
}

\author{
Estimation of the expected genetic gain of Pinus tecunumanii \\ in forest plantations in Oxapampa, Peru
}

\author{
Guillermo E. Gorbitz ${ }^{1}$, Lino Ríos ${ }^{1}$, Carlos Marujo ${ }^{1}$, \\ Víctor Cornejo ${ }^{2}$, Ray Medina ${ }^{3}$ y Lyanna Sáenz ${ }^{1, *}$
}

Recibido: 20 agosto 2020 | Aceptado: 20 noviembre 2020 | Publicado en línea: 18 diciembre 2020 Citación: Gorbitz, GE; Ríos, L; Marujo, C; Cornejo, V; Medina, R; Sáenz, L. 2020. Estimación de la ganancia genética esperada de Pinus tecunumanii en plantaciones forestales en Oxapampa, Perú. Revista Forestal del Perú 35 (3, Número Especial): 56-64. DOI: http://dx.doi.org/10.21704/ rfp.v35i3.1601

\begin{abstract}
Resumen
En el departamento de Pasco (Oxapampa) el Pinus tecunumanii F. Schwerdtf. Ex Eguiluz \& J.P. Perry es la especie forestal más plantada por su adaptación, calidad de madera y valor en el mercado nacional. El objetivo de este trabajo fue identificar árboles plus y estimar la ganancia genética esperada en plantaciones forestales de 7, 11 y 12 años de edad en 50 ha. La selección de los árboles se basó en la evaluación fenotípica del árbol candidato y sus cuatro mejores vecinos en un radio de 15 $\mathrm{m}$, evaluando individualmente las primeras cuatro trozas de 2,5 $\mathrm{m}$ de largo. Los arboles seleccionados se clasificaron en lista A cuando superaron en volumen y calidad a sus cuatro mejores vecinos, y en lista $B$, aquellos que superaron una variable sea volumen o calidad. La ganancia genética (GG) se estimó mediante el producto del diferencial de selección x heredabilidad. Además, se calculó el índice de selección, siendo de 1 árbol cada 1,06 ha. De 47 árboles seleccionados, 15 fueron clasificados en la lista A y 12 en la lista B. Al seleccionar y clonar los individuos de la lista A, se esperan GG de $12,66 \%$ en volumen, 7,26\% en calidad y para el caso de propagación por semillas GG de 10,55\% en volumen y $6,23 \%$ en calidad. Los resultados sugieren un progreso genético importante que permitirá elevar la productividad y sostenibilidad en la producción de semilla de calidad genética superior, para futuras plantaciones comerciales de Oxapampa, sin embargo, se recomienda ampliar la base genética y comprobar los resultados mediante ensayos en diferentes sitios potenciales.
\end{abstract}

Palabras clave: Árbol plus, selección diferencial, fenotipos, mejoramiento de árboles

\footnotetext{
${ }^{1}$ Dipteryx SAC, Pucallpa, Perú

${ }^{2}$ Universidad Nacional Agraria La Molina, Av. La Molina s/n, La Molina, Lima, Perú

${ }^{3}$ Investigador independiente

* Autor de Correspondencia: hellen@dipteryx.org
} 


\begin{abstract}
In the department of Pasco (Oxapampa) the Pinus tecunumanii F. Schwerdtf. Former Eguiluz \& J.P. Perry is the most widely planted forest species for its adaptation, wood quality and value in the national market. The objective of this work was to identify plus trees and estimate the expected genetic gain in forest plantations of 7, 11 and 12 years of age in 50 ha. The selection of the trees was based on the phenotypic evaluation of the candidate tree and its four best neighbors in a radius of $15 \mathrm{~m}$, individually evaluating the first four $2.5 \mathrm{~m}$ long logs. The selected trees were classified in list A when they exceeded their four best neighbors in volume and quality, and in list B, those that exceeded a variable, either volume or quality. Genetic gain $(G G)$ was estimated by the product of the selection differential $\mathrm{x}$ heritability. In addition, the selection index was calculated, being 1 tree per 1.06 ha. Of 47 selected trees, 15 were classified in list A and 12 in list B. When selecting and cloning the individuals from list A, GG of $12.66 \%$ in volume, $7.26 \%$ in quality and for the case of propagation by GG seeds of $10.55 \%$ in volume and $6.23 \%$ in quality. The results suggest an important genetic progress that will allow to increase the productivity and sustainability in the production of seed of superior genetic quality, for future commercial Oxapampa plantations, however, it is recommended to broaden the genetic base and verify the results through trials in different potential sites.
\end{abstract}

Key words: Tree plus, differential selection, phenotypes, tree improvement

\section{Introducción}

Perú cuenta con 13 millones de hectáreas, aproximadamente, con aptitud forestal (CIFOR 2017) de las cuales en el departamento de Pasco existen 7118.69 ha de plantaciones forestales (nativas e introducidas) (SERFOR 2020). Entre las especies introducidas plantadas para la producción de madera, con fines comerciales, se encuentran el eucalipto (Eucalyptus spp.) y pino (Pinus spp.), que proveen madera de alta calidad y de demanda en el mercado internacional (CIFOR 2017). La especie Pinus tecunumanii F. Schwerdtf. ex Eguiluz \& J.P. Perry, presenta rápido crecimiento, mayor densidad y mejor resistencia a la sequía (Cardoso y de la Torre 2016), siendo la más plantada en la provincia de Oxapampa (Perú), ocupando un área aproximada de 4000 ha (PRODEFAP 2016).

La actividad forestal a través del establecimiento de plantaciones se viene incrementado significativamente, debido principalmente a la escasez de madera del bosque natural y la creciente demanda de productos forestales, sin embargo, para convertir la actividad forestal en un proceso productivo rentable y seguro es necesario desarrollar programas de mejoramiento y de manejo, que conduzcan a la obtención de materia prima de la más alta calidad, con el menor costo posible (Murillo y Badilla 2004). Los principales problemas para la producción forestal en Perú radican en: a) bajo rendimiento; b) escasez y uso de semilla (sexual o asexual) de baja calidad genética; c) d) ausencia de un programa de mejoramiento genético sexual y clonal (CIFOR 2017).

El éxito de un programa de mejoramiento genético (PMG) depende de la intensidad de selección (exigencia) de los árboles parentales. Las ganancias esperadas derivan, tanto del control genético de las características de interés como de la variabilidad existente en la población (Zobel y Talbert 1988; Balcorta y Vargas 2004). El diferencial de selección es útil para predecir la respuesta de la selección en especies forestales (Zobel y Talbert 1988), y está altamente correlacionado con la ganancia genética, que es el fin de un programa de mejoramiento genético (Balcorta y Vargas 2004). Las estimaciones de ganancia genética esperada, le permiten al mejorador forestal conocer su progreso genético potencial y decidir, respecto los individuos que componen la población comercial y cuál es la población de mejoramiento (Vallejos et al. 2010). En este contexto, el objetivo de este trabajo fue identificar árboles plus y estimar la ganancia genética esperada en plantaciones forestales, con el propósito de 
generar una fuente semillera local e iniciar un programa de mejoramiento genético, para las condiciones forestales de Oxapampa.

\section{Materiales y métodos}

\section{Área de estudio}

El estudio se realizó en plantaciones comerciales de Pinus tecunumanii del distrito de Huancabamba, provincia de Oxapampa, región Pasco. Localizado geográficamente a latitud: $10^{\circ} 35^{\prime} 39^{\prime \prime}$ y longitud: $75^{\circ} 23^{\prime} 03^{\prime \prime}$ y altitud de $1850 \mathrm{msnm}$. La temperatura y humedad relativa media anual es de $18,4^{\circ} \mathrm{C}$ y $86,0 \%$ respectivamente. La precipitación media anual es de $1411 \mathrm{~mm}$ (SENAMHI 2016). Así mismo, se encuentra en la zona de vida de bosque húmedo montañoso (Brack 1984, citado por López 2020). Los suelos en la zona de estudio son profundos, sin embargo, algunas áreas presentan suelos superficiales. La capa arable de la mayoría de las laderas exhibe aceptables propiedades físicas y en algunos casos alto nivel de materia orgánica, pero predominan los suelos de fertilidad natural baja (Gálvez 2003, citado por López 2020).

\section{Pre selección y selección de árboles plus}

El estudio inició con la pre selección de árboles plus, es decir, la selección de los árboles candidatos a árboles plus, para ello, se realizó una evaluación visual mediante un recorrido por los lotes de la plantación forestal de 7, 11 y 12 años de edad, las cuales sumaron un total de 50 hectáreas.

La identificación de individuos sobresalientes en las diferentes condiciones de suelo fue realizada utilizando la metodología propuesta por Zobel y Talbert (1984) adaptada por Vallejos et al. (2010). Esta metodología ha sido mejorada en Costa Rica, y supera las limitaciones de las metodologías tradicionales, cuando existen diferencias en edad y en calidad de sitio, entre los árboles seleccionados e, incorpora, la calidad del fuste y un índice de selección, como nuevos caracteres importantes desde el punto de vista económico (Murillo y Badilla 2004). También es importante tener cuidado con la selección de árboles plus en plantaciones de mucha edad. Debido a que imposibilita detectar caracteres importantes, que se expresan a temprana edad, como es el caso de las ramas (Vallejos et al. 2010). Todos los árboles pre-seleccionados fueron georreferenciados y posteriormente evaluados fenotípicamente respecto a sus cuatro mejores vecinos en un radio de 15 $m$, considerando los siguientes atributos descritos: a) posición sociológica, b) bifurcación, c) forma de fuste, d) ramas gruesas, e) ramas en repetición, f) forma de copa, g) estado fitosanitario, h) calidad de fuste (CALI), i) altura comercial (hCOM), j) volumen comercial (VolCOM), y k) diámetro a la altura del pecho (DAP). Los árboles pre-seleccionados que lograron superar la fase de verificación, se constituyeron como árboles plus "seleccionados", y pasaron a conformar la población base de la fuente semillera. Después se procedió a estimar el VolCOM, utilizando la fórmula de conicidad que incorpora el DAP y la hCOM, mediante la siguiente fórmula:

$$
\operatorname{VolCOM}=\left(\frac{D A P}{100}\right)^{2} \times 0,7854 \times h C O M \times 0,65
$$

La CALI, se estimó con el promedio ponderado de las primeras cuatro trozas comerciales de 2,5 m de largo cada una, con base a una escala de 1 a 4, donde el valor de 1 es la mejor calidad posible y un valor de 4 se asigna para trozas sin valor como madera sólida, propuestos por Murillo y Badilla (2004).

CALI $=$ Troza $1 \times 0,4+$ Troza $2 \times 0,3+$ Troza 3 $\times 0,2+\operatorname{Troza} 4 \times 0,1$

$$
\begin{aligned}
\text { Troza } 1 & =40 \%(0 \text { a } 2,5 \mathrm{~m}) \\
\text { Troza } 2 & =30 \%(2,51 \text { a } 5,0 \mathrm{~m}) \\
\text { Troza } 3 & =20 \%(5,01 \text { a } 7,5 \mathrm{~m}) \\
\text { Troza } 4 & =10 \%(7,51 \text { a } 10,0 \mathrm{~m})
\end{aligned}
$$

La variable calidad de fuste, se transformó a una escala de 1 a $100 \%$, para facilitar su comprensión e interpretación, de la siguiente manera:

$$
\mathrm{CALI}_{\text {invertida }}=100 \times\left(1-\left[\frac{\text { (calidad del árbol-1) }}{3}\right]\right)
$$


Los árboles seleccionados permitieron estimar la intensidad de selección i, versus la población total original de individuos de la plantación. Además, para efectos estadísticos, de cada árbol seleccionado se obtiene el diferencial de selección (S) en VolCOM y CALI con respecto a la media de los 4-5 mejores vecinos. La transformación porcentual de cada variable se obtiene por medio de la siguiente formula:

$$
\mathrm{S}(\%)=\frac{(\text { árbol seleccionado }- \text { promedio vecinos })}{\text { promedio vecinos }} \times 100
$$

Estos resultados de diferencial de selección (S) permite clasificar a los árboles seleccionados en 2 listas A y B. En A se incluyeron aquellos que presentan superioridad marcada con relación a todos sus mejores vecinos, tanto en VolCOM como en CALI, estos árboles conformarán la población comercial. Mientras que $\mathrm{B}$, comprendió los árboles que registran superioridad fenotípica solamente en 1 de los 2 caracteres (VolCOM ó CALI) respecto a sus cuatro mejores vecinos. Estos árboles, se asumen que poseen al menos un carácter deseable, que podría ser capturado a través de cruzamientos controlados en programas de mejoramiento.

\section{Ganancia genética}

Posteriormente con la información de (S), pudo estimarse la ganancia genética (GG) esperada para los árboles plus de la lista $\mathrm{A}$, a través de la siguiente fórmula (Zobel y Talbert 1984):

$\mathrm{GG}=\mathrm{S}($ diferencial de selección $\%) \times \mathrm{h}^{2}$ (heredabilidad en sentido amplio)

Donde: $\mathrm{h}_{\text {calidad }}^{2}=0,35 \mathrm{y} \mathrm{h}_{\text {volumen }}^{2}=0,30$ en "heredabilidad en sentido amplio", $\mathrm{y} \mathrm{h}_{\text {calidad }}^{2}=$ $0,30 \mathrm{y} \mathrm{h}_{\text {volumen }}^{2}=0,25$ en "heredabilidad en sentido estrecho" (Cornelius, 1994; Murillo y Badilla 2004).

En ese sentido, remplazando los valores de h2 calculamos la GG para volumen (GGv) y calidad (GGc) de los árboles plus. Todos los cálculos estadísticos fueron realizados con ayuda del programa Microsoft Excel.

\section{Resultados}

\section{Pre selección y selección de árboles plus}

El proceso de pre-selección realizado en las 50 ha de plantaciones de Pinus tecunumanii de 7, 11 y 12 años de edad, permitió identificar un total de 47 árboles candidatos, por sus características fenotípicas sobresalientes, en base a dos criterios fundamentales: volumen y calidad del árbol. De los árboles pre-seleccionados un $42,5 \%$ son inferiores y $57,8 \%$ registraron un rendimiento superior, correspondiendo a un total de 27 árboles seleccionados. En base a los árboles seleccionados se estimó la intensidad de selección que osciló entre un árbol por cada 2058 árboles evaluados, que equivale aproximadamente a un árbol por cada 1,58 ha, esto demuestra el rigor y exigencia en la selección de los árboles. Para efectos estadísticos, de los 27 árboles seleccionados respecto a la media de los cuatro mejores vecinos, se clasificaron en dos listas: aquellos que presentan superioridad marcada en volumen y calidad clasificados como árboles plus de la lista $\mathrm{A}$, permitieron identificar los 15 individuos que conformarán la población comercial. Mientras que aquellos individuos que no superen a sus vecinos en alguna de las dos variables, se clasifican en la lista $\mathrm{B}$, siendo 12 árboles. Se asume que presentan al menos un carácter deseable, el cual podría ser capturado a través de cruzas controladas en programas de mejoramiento. Estos árboles no se incorporan a la población comercial, sino que son fuentes de reserva para su utilización en los cruzamientos controlados.

En el Cuadro 1 se muestra el resultado de la evaluación de la lista $\mathrm{A}$, respecto a sus mejores cuatro vecinos. Puede notarse que únicamente 15 árboles son potenciales para usar a escala comercial. En estos casos se debe seleccionar más arboles plus para evitar problemas ligados a una base genética, o en todo caso intercambiar material genético. La utilización de estos árboles plus de $P$. tecunumanii como clones, registra un diferencial de selección de 42,20\% en volumen comercial y $20,75 \%$ en calidad. Además, se observa que el árbol plus $\mathrm{C} 13$ fue el de mayor volumen, con $1,36 \mathrm{~m}^{3}$ y su calidad de fuste resultó ser de 100\%. 
Estimación de la ganancia genética esperada de Pinus tecunumani en Oxapampa, Perú

\begin{tabular}{|c|c|c|c|c|c|c|c|}
\hline \multirow{2}{*}{ Árbol } & \multirow{2}{*}{$\begin{array}{c}\text { Edad } \\
\text { (años) }\end{array}$} & \multicolumn{2}{|c|}{ Árboles plus A } & \multicolumn{2}{|c|}{ Mejores vecinos } & \multicolumn{2}{|c|}{ Diferencial* } \\
\hline & & $\begin{array}{c}\text { Volumen } \\
\left(\mathbf{m}^{3}\right)\end{array}$ & $\begin{array}{c}\text { Calidad } \\
(\%)\end{array}$ & $\begin{array}{c}\text { Volumen } \\
\left(\mathrm{m}^{3}\right)\end{array}$ & $\begin{array}{c}\text { Calidad } \\
(\%)\end{array}$ & $\begin{array}{c}\text { Volumen } \\
(\%)\end{array}$ & $\begin{array}{c}\text { Calidad } \\
(\%)\end{array}$ \\
\hline $\mathrm{C} 13$ & 12 & 1,36 & 100,00 & 0,91 & 96,67 & 48,52 & 3,44 \\
\hline $\mathrm{C} 16$ & 12 & 0,89 & 100,00 & 0,79 & 82,50 & 12,34 & 21,21 \\
\hline $\mathrm{C} 20$ & 12 & 0,70 & 86,67 & 0,53 & 71,67 & 32,39 & 20,93 \\
\hline $\mathrm{C} 30$ & 12 & 1,08 & 100,00 & 0,79 & 92,50 & 37,48 & 8,11 \\
\hline $\mathrm{C} 34$ & 12 & 1,12 & 100,00 & 0,81 & 78,30 & 37,59 & 27,71 \\
\hline $\mathrm{C} 36$ & 12 & 0,87 & 100,00 & 0,51 & 85,83 & 71,56 & 16,51 \\
\hline $\mathrm{C} 37$ & 11 & 1,14 & 96,70 & 0,73 & 77,50 & 56,03 & 24,77 \\
\hline $\mathrm{C} 38$ & 11 & 0,92 & 86,70 & 0,72 & 81,66 & 28,11 & 6,17 \\
\hline C39 & 11 & 0,85 & 100,00 & 0,50 & 75,00 & 69,57 & 33,33 \\
\hline $\mathrm{C} 40$ & 11 & 0,89 & 100,00 & 0,69 & 100,00 & 29,81 & 0,00 \\
\hline $\mathrm{C} 41$ & 11 & 1,05 & 100,00 & 0,71 & 81,66 & 46,62 & 22,46 \\
\hline $\mathrm{C} 42$ & 11 & 0,99 & 100,00 & 0,72 & 67,50 & 38,10 & 48,15 \\
\hline $\mathrm{C} 43$ & 11 & 0,80 & 96,70 & 0,61 & 80,00 & 30,93 & 20,88 \\
\hline $\mathrm{C} 46$ & 11 & 1,06 & 96,70 & 0,61 & 66,66 & 74,13 & 45,06 \\
\hline $\mathrm{C} 47$ & 11 & 0,85 & 90,00 & 0,71 & 80,00 & 19,83 & 12,50 \\
\hline
\end{tabular}

* Diferencial de selección en volumen $42,20 \%$ y calidad $20,75 \%$

Cuadro 1. Resultado de los atributos fenotípicos y diferencial de los árboles plus y sus vecinos seleccionados en la lista A de Pinus tecunumanii.

Por otro lado, en el Cuadro 2 se presentan los resultados de los 12 árboles seleccionados y clasificados en la lista $\mathrm{B}$, que registraron un diferencial de selección de $26,70 \%$ en volumen comercial y 5,06\% en calidad. Asimismo, el árbol C35 registró $1,12 \mathrm{~m}^{3}$ de madera comercial, que supera a sus vecinos $\left(0,60 \mathrm{~m}^{3}\right)$ en más de un $110 \%$. Este es un buen ejemplo de un árbol interesante para un programa de mejoramiento genético. Este tipo de árboles serían claramente eliminados de un programa convencional de mejoramiento genético. Sin embargo, exhibe un extraordinario volumen, criterio de importancia productiva y altamente deseable, motivo por el cual, son clasificados en la lista B. No debe olvidarse, que en la mayoría de los casos los caracteres de volumen y los cualitativos se manifiestan de manera independiente. El ár- bol ideal, superior en volumen y en calidad, es complicado de encontrar, pero es posible crearlo a través de cruzamientos controlados con progenitores sobresalientes.

\section{Ganancia genética}

Con la información obtenida de diferencial de selección de los árboles plus puede estimarse los resultados de GG. Teniendo en cuenta, que estos valores son por lo general más bajos de lo real, debido a que cada árbol plus fue evaluado contra sus mejores cuatro vecinos. Por lo tanto, el diferencial de selección registrado con este procedimiento, puede considerarse como moderado. En ese sentido, si se asume que los árboles plus de la lista $\mathrm{A}$, serán clonados directamente podrán lograr un impacto inmediato en la calidad y volumen por hectárea en las 
plantaciones, entonces la GG esperada podría estimarse como sigue (en sentido amplio):

$$
\mathrm{GG}=\mathrm{S}^{\star} \mathrm{h}^{2}
$$

$$
\begin{aligned}
\mathrm{GG}_{\text {volumen }} & =42,20 \% \times 0,30=12,66 \% \\
\mathrm{GG}_{\text {calidad }} & =20,75 \% \times 0,35=7,26 \%
\end{aligned}
$$

En el caso de trabajar en sentido estrecho, es decir, si el árbol plus no se logra clonar, se procede a colectar su semilla. En este caso la estimación de su ganancia genética será inferior, estimándose como sigue para la lista $\mathrm{A}$ :

$$
\begin{aligned}
\mathrm{GG}_{\text {volumen }} & =42,20 \% \times 0,25=10,55 \% \\
\mathrm{GG}_{\text {calidad }} & =20,75 \% \times 0,30=6,23 \%
\end{aligned}
$$

\section{Discusión}

La intensidad de selección de los árboles de Pinus tecunumanii preseleccionados para el tamaño de la plantación forestal fue de un árbol cada 2058 árboles. Esta intensidad de se- lección resulto ser superior a las recomendadas por (Zobel y Talbert 1984), quienes sugieren un árbol por cada 1000 individuos para huertos semilleros. De igual forma Balcorta y Vargas (2004), en México seleccionaron un árbol plus por cada 1111 individuos en la especie de Gmelina arborea Roxb. ex Sm. (Lamiaceae), corroborando que la intensidad de selección de los árboles plus A en Pinus tecunumanii resulto ser más exigente. Estos resultados demuestran el riguroso proceso de identificación y selección realizado en los árboles de Pinus tecunumanii permitiendo obtener un importante progreso genético. Los valores de intensidad de selección obtenidos se consideran suficientes para el comienzo de un programa de mejoramiento genético, ya que, permitió identificar un número importante de árboles plus de potencial genético alto, esto permitirá iniciar un programa de mejoramiento genético.

Los resultados de diferencial de selección calculados en esta investigación en árboles plus de la lista A de Pinus tecunumanii no superó a los reportados en Gmelina arborea de 51 y $76 \%$

\begin{tabular}{|c|c|c|c|c|c|c|c|}
\hline \multirow{2}{*}{ Árbol } & \multirow{2}{*}{$\begin{array}{c}\text { Edad } \\
(\mathbf{a n ̃ o s})\end{array}$} & \multicolumn{2}{|c|}{ Árboles plus B } & \multicolumn{2}{c|}{ Mejores vecinos } & \multicolumn{2}{c|}{ Diferencial* } \\
\cline { 3 - 8 } & & $\begin{array}{c}\text { Volumen } \\
\mathbf{( m}^{3} \mathbf{)}\end{array}$ & $\begin{array}{c}\text { Calidad } \\
\mathbf{( \% )}\end{array}$ & $\begin{array}{c}\text { Volumen } \\
\mathbf{( m}^{3} \mathbf{)}\end{array}$ & $\begin{array}{c}\text { Calidad } \\
\mathbf{( \% )}\end{array}$ & $\begin{array}{c}\text { Volumen } \\
\mathbf{( \% )}\end{array}$ & $\begin{array}{c}\text { Calidad } \\
\mathbf{( \% )}\end{array}$ \\
\hline C10 & 12 & 0,94 & 100,00 & 0,92 & 83,33 & 2,17 & 20,00 \\
\hline C11 & 12 & 1,02 & 86,67 & 0,88 & 85,00 & 16,34 & 1,96 \\
\hline C14 & 12 & 0,84 & 86,67 & 0,67 & 86,67 & 25,20 & 0,00 \\
\hline C18 & 12 & 0,98 & 86,67 & 0,74 & 82,50 & 32,88 & 5,05 \\
\hline C23 & 12 & 0,74 & 100,00 & 0,70 & 85,00 & 5,01 & 17,65 \\
\hline C28 & 12 & 0,91 & 76,67 & 0,72 & 82,50 & 26,75 & $-7,07$ \\
\hline C29 & 12 & 0,95 & 100,00 & 0,96 & 78,33 & $-1,07$ & 27,67 \\
\hline C31 & 12 & 0,97 & 93,33 & 0,83 & 92,50 & 16,04 & 0,90 \\
\hline C32 & 12 & 0,95 & 90,00 & 0,80 & 90,00 & 18,59 & 0,00 \\
\hline C35 & 12 & 1,12 & 86,70 & 0,60 & 96,66 & 85,33 & $-10,30$ \\
\hline C44 & 11 & 1,05 & 83,30 & 0,58 & 81,66 & 79,28 & 2,01 \\
\hline C45 & 11 & 0,83 & 90,00 & 0,73 & 87,50 & 14,17 & 2,86 \\
\hline
\end{tabular}

*Diferencial de selección en volumen $26,70 \%$ y calidad $5,06 \%$

Cuadro 2. Características de los atributos fenotípicos y diferencial de los árboles plus y sus vecinos seleccionados en la lista B de Pinus tecunumanii. 
Estimación de la ganancia genética esperada de

en diferencial de volumen comercial y calidad (Espitia et al. 2016). La misma tendencia en el diferencial de selección se pudo observar a lo reportado por Kumar et al. (2006), quienes reportaron valores de $40 \%$ para calidad. Sin embargo, nuestros resultados fueron superiores a los reportados por Balcorta y Vargas (2004), quienes registraron valores de $40 \%$ para volumen comercial, de igual forma Vallejos et al. (2010), reportan resultados en Tectona grandis (Lamiaceae) de $22,88 \%$, en volumen, y $21,83 \%$ en calidad. A pesar de lo anterior, se debe tener presente, que estos valores de diferencial de selección (S) son por lo general más bajos de lo real, debido a que cada árbol plus fue evaluado contra sus mejores cuatro vecinos. La explicación es que estos vecinos son competidores muy fuertes $y$, por tanto, no representativos del material ordinario (sin mejoramiento) que usualmente se obtiene de los viveros comerciales. Por tanto, el valor del diferencial de selección podría ser inclusive aún mayor superando significativamente a la población base (Vallejos et al. 2010). Estos resultados evidencian que, para una primera etapa de un programa de mejoramiento genético, estos valores son adecuados y conservadores, lo cual es de suma importancia para la toma de decisiones futuras y estrategias de mejoramiento.

Finalmente se estimó los resultados de ganancia genética esperada (GGE) para los árboles plus de la lista A y de la lista B, tanto para volumen y calidad. Los resultados registrados son menores a los reportados por varios autores; como Vallejos et al. (2010), quienes relacionan ganancias genéticas esperadas entre 20-25\% en volumen, o asimismo Rojas y Arias (2004), quienes reportan en Pinus caribaea var. hondurensis Barr. ganancias del $23 \%$ en volumen. Por su parte, Mesen (2001), estimó ganancias genéticas en Gmelina arborea del 17\% en altura y $43 \%$ en volumen; y Espitia et al. (2016), para la misma especie reportaron ganancias genéticas en árboles plus A de 51 y $76 \%$ en volumen comercial y calidad del fuste respectivamente. Sin embargo, si se tiene en cuenta que, con incrementos del $4 \%$ por conceptos de ganancias genéticas en volumen se cubren los costos de un programa de mejoramiento genético fores- tal y se recupera la inversión realizada (Ipinza 1998, Cornelius y Ugarte Guerra 2010), los resultados obtenidos en esta investigación permiten deducir una alta rentabilidad económica para el programa de mejoramiento que se realiza en Oxapampa.

Ello a su vez, facilita contar con material vegetal para clonar los mejores árboles. De acuerdo con Murillo y Badilla (2009), los arboles producidos a partir de clonación presentan una mayor ganancia genética debido a que capturan el $100 \%$ de la información genética, con esta información se puede argumentar que los árboles plus A seleccionados, producirán árboles con mayores características de crecimiento y mejor calidad del fuste en las siguientes generaciones. Lo cual permitirá aumentar la productividad y la calidad de la materia prima, beneficiando al productor de madera y la industria. Por lo tanto, este estudio contribuirá significativamente con hacer más sostenible el negocio forestal en el distrito de Oxapampa en el largo plazo, debido a que Pinus tecunumanii es una de las especies forestales que más se planta para la producción de madera.

De esta manera con los resultados de las evaluaciones se obtuvieron que 15 árboles (55,5\%) fueron clasificados como árboles plus A, los mismos que, por sus características pueden ser utilizados como progenitores para siembras comerciales, utilizando su semilla sexual o clonándolas directamente con la finalidad de obtener mayor ganancia genética potencial (Ipinza 1998, Mesen 2001, Murillo y Badilla 2009, Vallejos et al. 2010). Mientras que los 12 árboles plus B (44,5\%) no se incorporarán a la población comercial, debido a que presentan una condición de superioridad solamente en uno de los dos caracteres volumen o calidad. Por lo tanto, se mantendrán a la espera de su utilización en los programas de mejoramiento controlado como señalan Vallejos et al. (2010).

\section{Conclusiones}

Se logró identificar 27 árboles sobresalientes de Pinus tecunumanii por sus características fenotípicas, de los cuales 15 fueron selecciona- 
dos en lista A para utilización inmediata en la población comercial y 12 árboles en la lista B para conformar parte de la población de mejoramiento. La ganancia genética esperada en volumen y calidad de los árboles de la lista $\mathrm{A}$, cuando clonadas y propagadas mediante semillas permitirá elevar la productividad y obtener semillas de calidad genética superior, para futuras plantaciones comerciales en Oxapampa.

\section{Agradecimientos}

El presente trabajo se ha realizado gracias al financiamiento del Programa de Desarrollo Forestal Sostenible, Inclusivo y Competitivo en la amazonia peruana del SERFOR.

\section{Bibliografía}

Brack, A. 1984. Plan Maestro del Parque Nacional Yanachaga Chemillén. USAID/PEPP. Informe. Oxapampa, Pasco. Lima, Perú.

Balcorta, H; Vargas, J. 2004. Variación fenotípica y selección de árboles en una plantación de melina (Gmelina arborea Linn., Roxb.) de tres años de edad. Revista Chapingo 10 (1):13-19.

Cardoso y de la Torre, J. 2016. Evaluación del crecimiento, productividad maderable y rendimiento en la introducción de especies de los géneros Pinus y Eucalyptus en la región Cusco. XII Congreso Nacional Forestal CONAFOR. $13 \mathrm{p}$.

CIFOR, (Centro para la Investigación Forestal Internacional). 2017. Las plantaciones forestales en Perú: Reflexiones, estatus actual y perspectivas a futuro. Indonesia, s.e. $40 \mathrm{p}$.

Cornelius, J; Ugarte Guerra, L. 2010. Introducción a la Genética y domesticación forestal para la Agroforestería y Silvicultura. Centro mundial para la agroforestería-ICRAF. 124 p.

Espitia, M; Murillo, O; Castillo, C. 2016. Ganancia genética esperada en melina (Gmelina arborea Roxb.) en córdoba (Colombia). Revista Arvore 40(1):71-80.

Ipinza, R. 1998. Mejoramiento genético forestal. Serie Tecnica $\mathrm{N}^{\circ} 42$, Programa CONIF- Minis- terio de Agricultura sobre investigaciones en semillas de especies forestales nativas. Santafé de Bogota, INSEFOR. p. 162.

López, J. 2020. Efecto de sustratos y fertilizantes en la optimización del crecimiento de plántulas de Pinus tecunumanii Schw. ex Eguiluz et Perry en condiciones de vivero. Tesis para optar el título de ingeniero forestal. Universidad Nacional Agraria La Molina.132 p.

Mesen, F. 2001. Introducción al mejoramiento genético forestal. En: Identificación, selección y manejo de fuentes semilleras. Serie Técnica / No. 32. Convenio CONIF, INSEFOR y MADR. Bogotá (Colombia), septiembre. 118 p.

Murillo, O.; Badilla, Y. 2009. Reproducción clonal de árboles. Taller de Publicaciones. Instituto Tecnológico de Costa Rica, ITCR. Cartago. $45 \mathrm{p}$.

Murillo, O; Badilla, Y. 2004. Evaluación de la calidad y estimación del valor en pie de la plantación forestal. Escuela de Ingeniería Forestal, ITCR. Cartago, Costa Rica. 50 p.

PRODEFAP (Programa de Desarrollo Forestal Sostenible, Inclusivo y Competitivo en la Amazonia Peruana). 2016. Estudio de las cadenas productivas y estrategias para la promoción de plantaciones forestales con fines comerciales y sostenibles en el Perú. 40 p.

Ramos, H. A. 2015. Propagación por estacas de bolaina blanca (Guazuma crinita Mart.) provenientes de árboles candidatos a plus en condiciones de cámara de sub-irrigación. Tesis para optar el título de ingeniero forestal. Universidad Nacional Agraria La Molina.132 p.

Rojas, F; Arias, D. 2004. Manual para productores de Melina (Gmelina arborea) en Costa Rica. Cartago (Costa Rica). 86 p.

SENAMHI (Servicio Nacional de Meteorología e Hidrología), 2016. Data histórica del distrito de Huancabamba (en línea). Consultado 5 ago. 2020. Disponible en http: //www.senamhi. gob.pe/mapas/mapaestaciones/ dat esta tipo. php? estaciones $=4725$ D79C.

SERFOR (Servicio Nacional Forestal y de Fauna Silvestre), 2020. SNIFFS - Componente Es- 
tadístico (en linea). Consultado 14 oct. 2020. Disponible en http://sniffs.serfor.gob.pe/estadistica/es/tableros/registros-nacionales/plantaciones.

Vallejos, J; Badilla, Y; Murillo, O. 2010. Metodología para la selección e incorporación de árboles plus en programas de mejoramiento genético forestal. Revista Agronomia Costarricense 34(1):105-119.

Vasquez, AG; Nogueira, AS; Kirchner, FF; Berger, R. 2007. Uma síntese da contribuição do gênero Pinus para o desenvolvimento sustentável no sul do Brasil. Floresta 37(3):445-450. DOI: https://doi.org/10.5380/rf.v37i3.9941.

Zobel, B; Talbert, J. 1984. Applied Forest Tree Improvement. John Wiley\& Sons. USA. 510 p.

Zobel, B; Talbert, J. 1988. Técnicas de mejoramiento genético de árboles forestales. Ed. Limusa. México D.F. 545p. 